\title{
Educação em saúde nas páginas da REBEn no perído de 1995 a 2005
}

\author{
Education in health on REBEn's pages in the period from 1995 to 2005
}

Educación en salud en las paginas de la REBEn en lo periodo del 1995 al 2005

\author{
Viviane Lemes da Silva Carvalho', Viviane de Queiroz Clementino', \\ Lícia Maria de Oliveira Pinho' \\ 'Universidade Católica de Goiás, Centro de Estudos em Enfermagem e Nutrição. Goiânia, GO
}

Submissão: 13/02/2007

Aprovação: 01/12/2007

\section{RESUMO}

Durante nossa graduação, vivenciamos diversas práticas de educação em saúde Que nos motivou realizar este estudo. Os objetivos são descrever as práticas educativas desenvolvidas por enfermeiros (as); identificar as pessoas, o local e descrever os resultados obtidos com as práticas educativas. Estudo do tipo bibliográfico sistematizado. As informações foram coletadas através da seleção e análise, dos artigos sobre educação em saúde, publicados na Revista Brasileira de Enfermagem (REBEn), no período de 1995 a 2005 . Após a análise, 38 artigos foram selecionados e agrupados. Pudemos observar Que as práticas educativas acontecem com maior freeüência na unidade hospitalar, as estratégias utilizadas têm sido muito criativas, abrangem usuários de várias faixas etárias e os enfermeiros são os Que mais desenvolvem praticas educativas.

Descritores: Educação em saúde; Aprendizagem; Papel do enfermeiro.

\section{ABSTRACT}

During our graduation, we live deeply many practical of education in health that motivated us to carry through this study. The objectives are to describe practical educative developed by nurses; identify the people, the place and describe the results gotten with the educative practical. Study of the systemize bibliographical type. The information had been collected through the election and analysis, of articles on education in health, published in the Brazilian Magazine of Nursing (REBEn), in the period of 1995 to 2005 . After the analysis, 38 articles had been selected and grouped. We could observe that the educative practical happen more frequently at the hospital, the used strategies have been very creative, they enclose users of all ages and the nurses are the ones that more develop educative practices.

Descriptors: Education in health, Learning, Nurse's role.

\section{RESUMEN}

Durante nuestra graduación, vivimos diferentes prácticas de educación en la salud Que nos ha motivado a hacer este estudio. Los objetivos son describir las practicas educativas desarrollados por las (os) enfermeras (os); identificar la gente, el lugar y describir los resultados prácticos conseguidos con las practicas educativas. Estudio del tipo bibliográfico de la sistematización. Las informaciones habían sido recogidas con la elección y el análisis, de artículos sobre la educación en salud, publicados en la Revista Brasileña del Oficio de Enfermera (REBEn), en el período de 1995-2005. después del análisis, 38 artículos habían sido seleccionados y agrupados. Podríamos observar que las practicas educativas suceden más con frecuencia en el hospital, las estrategias usadas han sido muy creativas, incluyen a usuarios e numerosas edad y las enfermeras son las Que más desenvolved las practicas educativas.

Descriptores: Educación en salud; Aprendizaje; Rol de la enfermera. 


\section{INTRODUÇÃO}

Educação em saúde é uma prática constante no cotidiano de um enfermeiro, principalmente para o que atua em saúde pública. Sabese Que as práticas educativas são inúmeras, porém, sua eficácia continua Questionada.

Essa pesquisa é resultado da nossa vivência prática na educação em saúde. Seu objetivo é aprofundar o nosso conhecimento no assunto, buscando conhecer as práticas educativas realizadas por enfermeiros(as).

Durante a nossa graduação, vivenciamos, em vários momentos, a prática em educação e saúde, mas de maneira obrigatória, imposta, tanto para nós, estudantes, como para os usuários do Sistema Único de Saúde Que se encontravam na unidade. O Que se passava aos pacientes era algo pronto e fechado, o Que gerava em nós constantes Questionamentos acerca da eficácia de tais ações e se seriam aquela a maneira mais correta de realizá-las.

A evolução da saúde está relacionada com a evolução da sociedade. As práticas educativas têm como objetivo instruir indivíduos e grupos para colaborarem na melhoria da saúde da população. Estas práticas estão presentes desde tempos remotos, Quando a maioria da população buscava a medicina popular para resolver seus problemas de saúde ${ }^{(1)}$.

A educação em saúde é enfatizada na Lei n ${ }^{\circ} 8.080$, de 19 de setembro de 1990. Ela regula as ações e os serviços de saúde em todo o território nacional, dispondo, no seu artigo $2^{\circ}$ Que: "a saúde é direito fundamental do ser humano, devendo o Estado prover as condições indispensáveis ao seu pleno exercício"(2). Além de determinar a saúde como direito básico de todos, enuncia também, no artigo $3^{\circ}$, Que: "A saúde tem como fatores determinantes e condicionantes, entre outros, a alimentação, a moradia, o saneamento básico, o meio ambiente, o trabalho, a renda, a educação, o transporte, o lazer e o acesso aos bens e serviços essenciais; os níveis de saúde da população expressam a organização social e econômica do País"(2).

Criado em 1994, o Programa de Saúde da Família (PSF) tem sido a porta de entrada para o atendimento do Sistema único de Saúde-SUS, e é regido por seus princípios: universalidade, integralidade e equidade. Isto exige dos profissionais uma nova dinâmica de atuação, Que compreenda o planejamento das ações em saúde a partir do conhecimento da realidade da população. As práticas educativas são uma das estratégias para se alcançar estes princípios. São realizadas através de palestras, visitas domiciliares, campanhas educativas, reuniões em grupos, e também de forma individual, através da consulta de enfermagem ${ }^{(3)}$.

Este trabalho se justifica pelo nosso interesse em conhecer os métodos usados pelos(as) enfermeiros(as) para desenvolver as práticas educativas, bem como os resultados obtidos. Desejamos atuar em Saúde Pública e aprofundar nossos estudos em educação e saúde após a graduação.

\section{Repensando "Educação em Saúde"}

A educação que hoje se desenvolve, tem sua base em princípios filosóficos. A maneira como se "faz" educação depende do momento histórico vivenciado pela sociedade. Mas o Que é educação? “... a educação enquanto processo histórico evolui à medida que a sociedade evolui. É um processo dinâmico e flexível, Que possibilita ao ser humano, diverso e singular, no âmbito individual e coletivo, o desenvolvimento de suas potencialidades, podendo atingir autonomia e decidir sobre seus objetivos e ações"(4).

Para se fazer educação é preciso conhecer a si mesmo, para poder compreender o outro, ter empatia, trocar conhecimento. Educar é um processo contínuo, em Que se ensina e aprende a cada dia, respeitando o saber do outro e aprendendo com ele. "Assim ensinar não é transferir conhecimentos, é sim criar possibilidades para sua construção e produção"(5).

Para se definir saúde, recorremos à Lei n ${ }^{\circ} 8.080 / 90$, denominada Lei Orgânica da Saúde, em seu artigo segundo, parágrafo primeiro: "O dever do Estado de garantir a saúde consiste na formulação e execução de políticas econômicas e sociais Que visem à redução de riscos de doenças e de outros agravos e no estabelecimento de condições Que assegurem acesso universal e igualitário às ações e aos serviços para a sua promoção, proteção e recuperação"(2). A saúde pode ser entendida como "...um processo dinâmico em Que o homem luta contra as forças Que tendem a alterar o equilíbrio da saúde; o complexo processo de redução da saúde não é provocado por fatores simples ou específicos, mas pelo resultado da ligação contínua entre causas e efeitos"(I).

Com o intuito de unir os unitermos referidos acima, buscamos uma definição para educação em saúde. Nas sociedades ocidentais e modernas, a Educação em Saúde realizada até então apoiou-se no modelo biomédico-mecanicista, nos resíduos do higienismo e na valorização da razão sobre o sensível ${ }^{(6)}$.

Profissionais e comunidade devem entender Que educação em saúde é uma prática oferecida pelos serviços de saúde e deve ser mantida e completada pela ação da população, através de seu conhecimento e interesse pelas práticas educativas de saúde. "A educação em saúde é uma ação básica de saúde importante Quando estiver baseada na reflexão crítica do grupo, porque o princípio dessa educação é o desenvolvimento da consciência critica das causas, dos problemas e das ações necessárias para a melhoria das condições"(I)

Com base nessas definições, neste trabalho, buscamos identificar as práticas educativas desenvolvidas pelos (as) enfermeiros (as), conhecendo os resultados obtidos em seus artigos. Observamos, desta forma, se a educação em saúde está instigando pensamentos críticos na população e, conseQüentemente, gerando transformação no indivíduo, aumentando a sua autonomia.

\section{OBJETIVOS}

- Descrever as práticas educativas desenvolvidas por enfermeiros(as);

- Identificar os sujeitos Que fazem parte do processo de educação em saúde;

- Identificar as estratégias usadas para desenvolver as práticas educativas;

- Identificar o local onde as práticas educativas são realizadas;

- Descrever os resultados obtidos com as práticas de educação em saúde.

\section{METODOLOGIA}

Trata-se de um estudo do tipo bibliográfico. A revisão de 
literatura proporciona aos leitores os antecedentes para a compreensão do conhecimento atual sobre um tópico e esclarece a importância do novo estudo. As revisões de literatura servem, assim, como função integradora e facilitam o acúmulo de conhecimentos"(7).

Assim, apresentamos as práticas educativas desenvolvidas por enfermeiros(as), identificando as pessoas Que fazem parte do processo de educação em saúde, bem como as estratégias usadas para desenvolver as práticas educativas, o local onde estas acontecem e os resultados obtidos com estas práticas de educação em saúde.

Os dados foram coletados na Revista Brasileira de Enfermagem (REBEn), no período de 1995 a 2005. A busca dos artigos foi iniciada tendo como critério seletivo os descritores: "enfermeiro(a)", "educação", "saúde" e "processo de aprendizagem”. Logo no princípio, notamos que este critério não seria adequado, visto Que, mesmo se tratando de educação em saúde, estes não eram indexados.

A opção, então, foi escolhermos os textos a partir da leitura de todos os artigos publicados no referido período e selecionarmos aQueles Que contemplavam os objetivos propostos, seguindo um roteiro semi-estruturado. Para melhor compreensão e visualização, agrupamos os dados encontrados e os apresentamos em um Quadro Que analisamos posteriormente.

\section{RESULTADOS E DISCUSSÃO}

Após a análise dos textos, 39 artigos foram selecionados e agrupados em forma de Quadro, para melhor compreensão das práticas educativas desenvolvidas. Analisamos os títulos dos artigos, a metodologia, o local de realização dos encontros, as estratégias, os Indivíduos participantes e os resultados encontrados. Todas as categorias foram extraídas diretamente dos textos, e, em alguns artigos, não pudemos identificar a metodologia utilizada, o local de encontro, as estratégias e os indivíduos participantes.

Pudemos observar, a partir da análise dos artigos, Que os títulos dos artigos, em sua grande maioria, explicitam a prática de educação em saúde Que será desenvolvida, Quando não, estão explicitadas ao longo do texto.

A metodologia mais empregada foi à Qualitativa do tipo relato de experiência. Apenas um artigo foi definido como QuantiQualitativo; outros dois foram recortes de dissertação.

Quanto ao local de realização do encontro, foi uma surpresa constatar Que tais práticas educativas acontecem com maior freeüência na atenção terciária (unidade hospitalar) e não na atenção primária (unidades de atenção básica a saúde da família, centros de saúde, escolas, universidades e comunidade), como esperávamos, em função dos programas de prevenção e, em especial, pela criação do Programa de Saúde da Família, Que tem como objetivo reorganizar a atenção básica e o modelo assistencial preconizado pelo Sistema Único de Saúde.

As estratégias utilizadas têm sido muito criativas, por exemplo: orientação à beira do leito, com a presença do familiar cuidador; atividade grupal para familiares cuidadores; consultas de enfermagem; palestras; aconselhamento; entrevista; visita domiciliaria; terapia natural; acupuntura; reflexologia; Questionários; grupo de gestantes; programa de rádio; reuniões semanais; atividades de lazer; oficinas de criatividade; discussão de temas básicos; planejamento de alta; utilização de jogo educativo; implantação da sistematização da assistência de enfermagem; grupo de auto-ajuda; expressões corporais; dramatização; vídeos; orientação no pós operatório; confecção de manual de orientação; interação enfermeiro-paciente; relaxamento; grupos para diminuir a ansiedade do paciente frente a internação; orientação pré-operatória; grupos para a promoção social; elaboração de instrumentos técnico-pedagógicos e teste dos mesmos pelo grupo; cuidados universais e terapêuticos durante a hospitalização do paciente.

As práticas educativas abrangem usuários de várias faixas etárias, são desenvolvidas mesmo Que atendam um único usuário e sempre Que possível à família do paciente estava envolvida nas ações realizadas.

As ações educativas desempenhadas nos processos de educação em saúde foram realizadas por enfermeiros, na sua maioria; em alguns casos houve a participação de outros profissionais da eQuipe de saúde como médicos, pedagogos, assistente social, técnico de enfermagem, auxiliar de enfermagem e acadêmicos de enfermagem, medicina, pedagogia e psicologia, visando uma melhor assistência ao usuário.

Dos 39 artigos analisados, apenas três relataram não terem alcançado os objetivos propostos. As dificuldades e obstáculos encontrados se deram, provavelmente, pela forma soberana com Que os profissionais fundamentaram o processo de educação em saúde. Os demais alcançaram seus objetivos de levar os usuários ao autocuidado, com alcance de um nível de bem-estar satisfatório. Quando o usuário, por algum motivo, não conseguia ser agente do seu cuidar, um familiar participava do processo educativo, com o objetivo de auxiliá-lo a se cuidar.

Ao realizar a busca dos artigos, encontramos um Que descreve o porquê dos profissionais do Programa de Saúde da Família não praticarem educação em saúde. Assim, ele foi separado para se comparar os resultados do referido artigo ao Que havia sido apurado nesta pesQuisa.

O artigo tem como título "Entendimento e prática de ações educativas de profissionais do Programa de Saúde da Família de São Sebastião-AL: destacando dificuldades”. É um trabalho baseado na monografia de Melo, um dos autores do estudo.

"O interesse e curiosidade em realizá-lo surgiram no desenvolvimento das atividades no Programa Saúde da Família naQuele município, onde se percebeu Que o atendimento à clientela ocupava todo o tempo dos profissionais e Que as metas a serem alcançadas obedecendo a critérios da Secretaria Municipal de Saúde do Município levavam a um trabalho exaustivo, não individualizado, o Qual vinha dificultando a execução de outras atividades de interação com a comunidade"(8).

"Percebeu-se, ainda, a escassez das ações educativas voltadas para a promoção e proteção de saúde, assim como a produção de materiais educativos voltados para a informação da população em geral, priorizando os aspectos preventivos da saúde bem como a adoção de hábitos de vida saudáveis" ${ }^{(8)}$. As palestras são as mais prejudicadas, pois o espaço físico disponibilizado para tais atividades são as salas de espera, Que são consideradas impróprias, devido a movimentação constante de pessoas e excesso de barulho. Entendese Que a não priorização das atividades educativas possa ser explicada pelo conceito que os profissionais tenham de educação em saúde, 


\begin{tabular}{|c|c|c|c|}
\hline Ano & $\begin{array}{l}\text { Número de artigos } \\
\text { encontrados }\end{array}$ & Local & Sujeitos participantes \\
\hline $1995^{(9-11)}$ & 03 & Unidade hospitalar: 03 & \multirow{4}{*}{$\begin{array}{l}\text { Pacientes submetidos a consulta oftalmológica, em tratamento com } \\
\text { método de Ilizarov, crianças hospitalizadas e enfermeiros(as) } \\
\text { Enfermeiros(as), pedagoga, acadêmicos de enfermagem, pacientes } \\
\text { portadores de incapacidade do aparelho locomotor, trinta } \\
\text { mulheres, adolescente de rua, adolescentes matriculados no curso } \\
\text { de patrulheirismo da Rocinha, oito pessoas com deficiência visual. }\end{array}$} \\
\hline \multirow[t]{3}{*}{$1996^{(12-17)}$} & \multirow[t]{3}{*}{06} & $\begin{array}{l}\text { Unidade hospitalar: } 02 \\
\text { Universidade } 03 \\
\text { Comunicade } 01\end{array}$ & \\
\hline & & Universidade: 03 & \\
\hline & & Comunidade: 0 I & \\
\hline \multirow[b]{2}{*}{$1997^{(18-21)}$} & \multirow[b]{2}{*}{04} & Unidade hospitalar: 03 & \multirow{2}{*}{$\begin{array}{l}\text { Pacientes Que receberam orientação pré-operatória, Quarenta } \\
\text { dois clientes do serviço de lombalgia, oito clientes da comunidade } \\
\text { oito pacientes psicóticos com dificuldade de expressão verbal } \\
\text { enfermeiros(as), médico ou ortopedista, }\end{array}$} \\
\hline & & Comitê Comunitário: 01 & \\
\hline \multirow{5}{*}{$1998^{(22-26)}$} & \multirow{5}{*}{05} & $\begin{array}{l}\text { Núcleo de apoio } \\
\text { psicossocial: } 01\end{array}$ & \multirow{5}{*}{$\begin{array}{l}\text { Um individuo com depressão crônica, familiares de pacientes } \\
\text { crônicos, paciente do sexo masculino com } 68 \text { anos, Quarenta e um } \\
\text { pacientes internados na clínica cirúrgica, enfermeiros(as), } \\
\text { acadêmicos de enfermagem e de psicologia }\end{array}$} \\
\hline & & $\begin{array}{l}\text { Núcleo de estudos e } \\
\text { domicilio: } 01\end{array}$ & \\
\hline & & Domicilio: 01 & \\
\hline & & Unidade hospitalar: 0I & \\
\hline & & Não relatado: 01 & \\
\hline \multirow[b]{3}{*}{$1999^{(27-31)}$} & \multirow[b]{3}{*}{05} & CAPS: 01 & \multirow{6}{*}{ 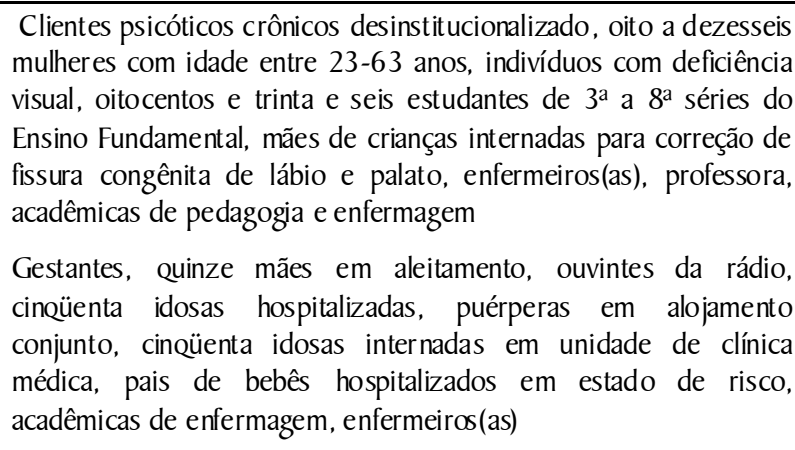 } \\
\hline & & Comunidade Rural: 01 & \\
\hline & & $\begin{array}{l}\text { Laboratório de } \\
\text { Enfermagem:01 } \\
\text { Escola: 01 } \\
\text { Unidade hospitalar: } 01\end{array}$ & \\
\hline \multirow[t]{3}{*}{$2000^{(32-38)}$} & \multirow[t]{3}{*}{07} & $\begin{array}{l}\text { Unidade básica de saúde: } \\
01\end{array}$ & \\
\hline & & Unidade hospitalar: 05 & \\
\hline & & Radio universitár ia: 01 & \\
\hline $200 I^{(39-40)}$ & 02 & $\begin{array}{l}\text { Clínica de enfermagem: } \\
01 \\
\text { Universidade: } 01\end{array}$ & $\begin{array}{l}\text { Enfermeiros(as), médicos, Quinhentos e vinte e seis acadêmicos de } \\
\text { enfermagem, trinta e dois acadêmicos de medicina }\end{array}$ \\
\hline \multirow{2}{*}{$2002^{(41-42)}$} & \multirow{2}{*}{02} & $\begin{array}{l}\text { Unidade Básica de } \\
\text { Saúde: } 01\end{array}$ & \multirow{2}{*}{$\begin{array}{l}\text { Dez participantes de um programa de educação em saúde para } \\
\text { diabetes, um paciente portador de miocardiopatia e a esposa do } \\
\text { mesmo, enfermeiros(as) }\end{array}$} \\
\hline & & $\begin{array}{l}\text { Unidade hospitalar e } \\
\text { domicilio: } 01\end{array}$ & \\
\hline \multirow[b]{2}{*}{$2003^{(43-44)}$} & \multirow[b]{2}{*}{02} & Unidade hospitalar: 0I & \multirow{2}{*}{$\begin{array}{l}\text { Cineüenta mulheres hipertensas, oito mulheres com HIV +, } \\
\text { enfermeira, médica, auxiliares de enfermagem e assistente social. }\end{array}$} \\
\hline & & $\begin{array}{l}\text { Centro municipal de } \\
\text { saúde: } 0 \text { I }\end{array}$ & \\
\hline $2004^{(45-46)}$ & 02 & $\begin{array}{l}\text { Unidade hospitalar: } 01 \\
\text { Escola: } 01\end{array}$ & $\begin{array}{l}\text { Dezoito clientes transplantados cardíacos, adolescentes de uma } \\
\text { escola pública, enfermeiras e acadêmicos de enfermagem. }\end{array}$ \\
\hline $2005^{(47)}$ & 01 & Unidade hospitalar: 01 & $\begin{array}{l}\text { Doze cuidadores familiares de nove idosos com acidente vascular } \\
\text { cerebral }\end{array}$ \\
\hline
\end{tabular}

Quadro 1. Número de produções por ano, local de realização e indivíduos participantes.

ou pela importância Que dão ao número de atendimentos impostos pela Secretaria Municipal de Saúde. O material relata também, não ter encontrado nenhum estudo Que tenha se preocupado em indagar até Que ponto a comunidade está interessada em ouvir palestras e Quais temas gostariam de discutir. Expressa, ainda, a dificuldade dos profissionais Quando são contratados para trabalhar numa Unidade de Saúde da Família, por terem uma formação desenvolvidas em Instituições de Saúde tradicionais, o Que vem sendo superado com os cursos e treinamentos.
"Este estudo também adQuire relevância Quando gera a possibilidade de se rever o patamar em Que o próprio PSF coloca a Questão da educação em saúde, em termos de prioridade, providenciando material e suporte pedagógico para a realização dessas atividades. E conseguiu apontar as principais reivindicações dos profissionais para a realização de ações educativas estimulantes: local apropriado para a realização de palestras; horário adeQuado para as palestras em Que boa parte da comunidade possa comparecer; estímulo à comunidade com material mais atraente, lanches, uso 
de recursos audiovisuais para além de cartazes; uma maior carga horária para desenvolver as ações preventivas nas Equipes de Saúde da Família; conscientização da população da importância de sua participação nas ações educativas; necessidade de transporte das equipes para o local das atividades educativas"(8).

"Os autores concluem que esta pesquisa revelou que a assistência à saúde, prestada no município de São Sebastião, está fortemente centrada na atenção curativa e no atendimento às Queixas do paciente e Que, embora os profissionais de saúde (médicos, enfermeiros e dentistas), tendo o conhecimento da importância das ações educativas para a comunidade onde trabalham, encontram dificuldades para desenvolver um processo educativo Que possa Ihes revelar a necessidade do saber viver uma vida saudável, do conhecer a doença e sua prevenção. O conjunto dos profissionais entrevistados assegura Que entendem e aceitam que possuem este compromisso, porém acreditam Que os gestores não estão dando a devida importância a esta parte do trabalho do PSF"(8).

Nos artigos analisados, os profissionais não relataram dificuldades como as expressas no artigo acima referido. Possivelmente pelo local onde as práticas educativas foram realizadas, sendo estas, em sua grande maioria, desenvolvidas nas unidades hospitalares, não exigindo do educador recursos didáticos, local adequado e busca do cliente na comunidade, pois este já se encontrava no hospital. Em alguns artigos não se exigia, ainda, do usuário mudanças de comportamento e reflexão sobre seus hábitos de vida. Percebemos, ainda, Que, mesmo com formação biomecanicista, os profissionais de saúde tinham interesse em desenvolver atividades de educação em saúde.

Ao analisar a produção anual acerca de educação em saúde, no período em estudo, notamos que houve um crescimento entre
1995 e 2000, sendo o ápice da produção o ano de 2000, com sete artigos publicados. No entanto, após este ano, inicia-se um declínio das publicações, chegando a ser publicado apenas um no ano de 2005, como mostra o Quadro I. Tal achado contradiz nossas expectativas, pois esperava-se Que, com a solidificação do PSF, as produções iriam aumentar, visto Que um dos princípios do Programa é promover a educação em saúde.

Salientamos, no entanto, Que a REBEn não tem como característica a concentração de publicações tematizadas.

\section{CONSIDERAÇÕES FINAIS}

Com base nos dados analisados notamos a pouca produção científica dos enfermeiros(as) Que atuam na rede básica de saúde. Os enfermeiros são os Que têm maior participação nas práticas educativas, e, se não as publicam, provavelmente isso se deve ao fato de não estarem vinculados à cursos de graduação e ou de pósgraduação, Que valorizam e exigem de seus docentes e discentes publicações anuais.

Consideramos importante o incentivo a estes profissionais, principalmente Quanto à publicação de suas práticas desenvolvidas no cotidiano de trabalho. Assim, pode-se conhecer e avaliar a eficácia das ações executadas. Cabe às Universidades, Que compartilham com seus acadêmicos os diversos campos de estágio, incentivar e propor projetos em parcerias com os profissionais da assistência.

Entendendo-se Que a educação em saúde é medida essencial na evolução da humanidade, e Que, portanto, faz-se necessário o incentivo e a adeQuação das práticas educativas, cabendo aos profissionais de nível superior a elaboração, coordenação e execução de tais ações.

\section{REFERÊNCIAS}

I. Kawamoto EE, Santos MCH, MattosTM. Enfermagem comunitária. São Paulo (SP): EPU; 1995.

2. Ministério da Saúde (BR). Conselho Nacional de Saúde. Lei 8.080 de 19/09/1990. Brasília (DF): Ministério da Saúde; 1990.

3. Castanho D, Almeida MH, Mottin LM, Tagliari MH. O SIAB como elemento para o planejamento das ações de saúde. Rev Técnico-Cient Enferm 2006; 4(14): 10-7.

4. Zampieri MFM. Vivenciando o processo educativo em enfermagem com gestantes de alto risco e seus acompanhantes. Texto Contexto Enferm 1999; 8(I): 203-2I.

5. Pinho LMO. Educação em saúde no Quotidiano do ser diabético [dissertação]. Belo Horizonte (MG): Universidade Federal de Minas Gerais; 1999.

6. Teixeira ER, Daher DV. Trabalhando com as representações dos sujeitos na educação em saúde. Texto Contexto Enferm 1999; 8(1): 312-25.

7. Polit DF, Beck CT, Hungler B. Fundamentos de Pesquisa em Enfermagem: métodos, avaliação e utilização. Porto Alegre (RS): Artmed; 2004.

8. Melo G, Santos RM, Trezza MCSF. Entendimento e prática de ações educativas de profissionais do Programa de Saúde da Família de São Sebastião-AL: detectando dificuldades. Rev Bras Enferm 2005; 58(3): 290-5.
9. Bertone AMC, Thomé S, Bachega MI. Assistência primária em saúde- atuação do enfermeiro frente as necessidades do portador de malformação congênita de lábio e/ou palato. Rev Bras Enferm 1995; 48(3): 204-11.

10. Cárnio AM, Cintra FA, Tonussi |AG. Orientação pré-operatória a pacientes com catarata e indicação de cirurgia ambulatorialrelato de experiência. Rev Bras Enferm 1995; 48(1): 39-45.

11. Tashiro MTO, Souza MF, Oliveira SD. Auto cuidado no tratamento pelo Método de Ilizarov- um estudo de caso. Rev Bras Enferm 1995; 48(1): 46-50.

12. Amorim MHC, Freitas ABS, Batista EM, Pacheco LN. Oficina de trabalho: "mulher-uma viagem ao seu corpo". Rev Bras Enferm 1996; 49(2): 28I-6.

13. Araújo EG, Nunes MMLG. Atos \& autores- o lúdico na educação em saúde. Rev Bras Enferm 1996; 49(3): 459-74.

14. Brum ZP, Pereira MA. Educação em saúde enfocando higiene, sexualidade e drogadição junto aos meninos de rua na faixa etária de II a 14 anos. Rev Bras Enferm 1996; 49(3): 33342.

15. Faria AB, Cruz ICF. Diagnóstico de enfermagem em cliente com (TCE) e em seu familiar e/ou pessoa significativa. Rev Bras Enferm 1996; 49(4): 549-68.

16. Ferolla EC, Lourenço C. Manual de Orientação sexual para o lesado medular metodologia de aplicação de resultados. Rev 
Bras Enferm 1996; 49(2): 165-82.

17. Pagliuca LMF, Costa EM, Costa NM, Sousa KM. Desenvolvimento tecnologia para prevenção e tratamento de emergências domésticas para cegos. Rev Bras Enferm 1996; 49(1): 83-104.

18. Bringuente MEO, Castro IS, Jesus JCG, Luciano LS. Fatores de risco para a coluna: avaliação em consulta de enfermagem. Rev Bras Enferm 1997; 50(3): 39 I-406.

19. Lima CB, BaptistaSS. Educação e Saúde visando à cidadania: práxis grupal de enfermeiras. Rev Bras Enferm 1997; 50(4): 469-76.

20. Tavare CM. Oficina de arte: atuação terapêutica da enfermeira psiQuiátrica. Rev Bras Enferm 1997; 50(4): 569-76.

21. Vale EG, Amorim MF, Freitas CB, Melo FP. Orientação préoperatória: análise compreensiva sob a ótica do cliente. Rev Bras Enferm 1997; 50(1): 31-6.

22. Albuquerque MCS, Cavalcante MSL. Relaxamento: uma estratégia no contexto da assistência de enfermagem. Rev Bras Enferm 1998; 51 (1): 35-52.

23. Rireiro V, Munari DB. Saúde mental em clientes cirúrgicos: o desenvolvimento de ações de enfermagem através do grupo de suporte/apoio. Rev Bras Enferm 1998; 5 I (1): 147-64.

24. Marcon SS, Rossini AFS, Aceti EL. Assistência de enfermagem domiciliar em equipe multiprofissional após o óbito do paciente. Rev Bras Enferm I 998; 5 I (3): 379-92.

25. Osinaga VLM, Scatena MC M; Rodrigues ARF. Enfermeira interagindo terapeuticamente com paciente idosa em depressão crônica. Rev Bras Enferm 1998; 5 I (2): 263-72.

26. Santos SSC. O cuidar da pessoa idosa no âmbito domiciliar: uma relação de ajuda na enfermagem. Rev Bras Enferm '998; 5 I (4): 665-76.

27. Martini JG, Gregis C, Jardim L. Gravidez na adolescência: da prática disciplinadora à pedagogia libertadora. Rev Bras Enferm 1999; 52(4): 539-46.

28. Mondini CCSD, Fontes CMB. Programa "mãe-participante". Rev Bras Enferm 1999; 52(1): 100-4.

29. Pagliuca LMF, Rodrigues IO. Métodos contraceptivos de barreira e DIU: tecnologia educativa para deficientes visuais. Rev Bras Enferm 1999; 52(3): 41 3-22.

30. Portella, Marilene Rodrigues. Cuidar para um envelhecer saudável: a construção de um processo educativo com mulheres rurais. Rev Bras Enferm 1999; 52(3): 355-64.

31. Ribas D L, Borenstein MS. CAPS-Florianópolis: uma experiência de grupo com clientes psicóticos fora dos muros do manicômio, durante dez anos. Rev Bras Enferm 1999; 52(2): 179-88.

32. Brunherotti MR, Pereira FL, Souza MI, Nogueira FS, Scochi CGS. Lazer para pais de bebês de risco: a experiência junto ao Hospital das Clínicas de Ribeirão Preto. Rev Bras Enferm 2000; 53(4): 435-42.

33. Cunha ICKO. Gente cuidando de gente: a arte do cuidar pelas ondas do radio. Rev Bras Enferm 2000; 53(3): 43 I-4.

34. Fonseca LMM, Scochi CGS, Bis CEF, Serra SOA. Utilizando a criatividade na educação em saúde em alojamento conjunto neonatal: opinião de puérperas sobre o uso de um jogo educativo. Rev Bras Enferm 2000; 53(2): 30 I - I0.

35. Marin MIS, Barbosa PMK, Takitane MT. Diagnóstico de enfermagem mais freqüentes entre idosas hospitalizadas em uma unidade de clínica médica e cirúrgica. Rev Bras Enferm 2000; 53(4): 513-23.

36. Marin MJS, Angerami ELS. Avaliação da satisfação de um grupo de idosas e cuidadores com o planejamento de alta. Rev Bras Enferm 2000; 53(2): 265-73.

37. Melo LL, Lima MADS. Mulheres no segundo e terceiro trimestres de gravidez: suas alterações psicológicas. Rev Bras Enferm 2000; 53(1): 81-6.

38. Rozário PS, Zagonel IPS. Proposta de cuidar com enfoøue educativo às mães em aleitamento materno. Rev Bras Enferm 2000; 53(3): 401-9.

39. Tashiro MTO, Orland R, Martins RCT, Santos E. Novas tendências terapêuticas de enfermagem - terapias naturais programa de atendimento. Rev Bras Enferm 200 I; 54(4).

40. Gomes VLO, Fonseca AD, Rodrigues MGS. Saúde oral: um desafio para a equipe de saúde. Rev Bras Enferm 2001; 54(1).

41. Freitas MC, Santana ME. Implementação da estratégia de ensino-aprendizagem à família de paciente crônico. Rev Bras Enferm 2002; 55(2): 146-50.

42. Penna CMM, Pinho LMO. A contramão dos programas de educação em saúde: estratégias de diabéticos. Rev Bras Enferm 2002; 55 I): 7-12.

43. Reis AL, Xavier IM. Mulher e AIDS: rompendo o silêncio de adesão. Rev Bras Enferm 2003; 56(I): 28-34.

44. Santos ZMSA, Silva RM. Consulta de enfermagem à mulher hipertensa: uma tecnologia para a educação em saúde. Rev Bras Enferm 2003; 56(6): 605-9.

45. Oliveira HM, Gonçalves MJF. Educação em saúde: uma experiência transformadora. Rev Bras Enferm 2004; 57(6): 76I-3.

46. Santos ZMSA, Oliveira VLM. Consulta de enfermagem ao cliente transplantado cardíaco- impacto das ações educativas em saúde. Rev Bras Enferm 2004; 57(6): 654-7.

47. César AM, Santos BRL. Percepção de cuidadores familiares sobre um programa de alta hospitalar. Rev Bras Enferm 2005; 58(6): 647-52 